\title{
Stem cell-based therapy for inflammatory bowel disease
}

\author{
Hiromichi Shimizu $^{1,2}$, Kohei Suzuki ${ }^{1,2}$, Mamoru Watanabe ${ }^{3}$, Ryuichi Okamoto ${ }^{4}$ \\ Departments of ${ }^{1}$ Gastroenterology and Hepatology, and ${ }^{2}$ Endoscopy, ${ }^{3}$ Institute of Advanced Study, ${ }^{4}$ Center for Stem Cell and Regenerative \\ Medicine, Tokyo Medical and Dental University, Tokyo, Japan
}

Inflammatory bowel disease (IBD) is an idiopathic, multi-etiological disease characterized by inflammation and mucosal destruction of the gastrointestinal tract. Despite the remarkable advance in immunomodulating therapies, there still remains a certain population of patients who are refractory to conventional as well as biologic therapies and fail to achieve mucosal healing. To improve the prognosis of those patients, at least 2 types of stem cells have been tested for their potential therapeutic use. Transplantation of hematopoietic stem cells or mesenchymal stem cells have been tested in several clinical studies, but their beneficial effect still remains controversial. In this review, we would like to overview the recent clinical challenges of stem cellbased therapies in IBD and also introduce our new therapeutic plan of intestinal stem cell transplantation for IBD, based on our ex vivo intestinal organoid culture technique. (Intest Res 2019;17:311-316)

Key Words: Mucosal healing; Hematopoietic stem cell; Mesenchymal stem cell; Intestinal stem cell; Organoids

\section{INTRODUCTION}

Inflammatory bowel disease (IBD) is a chronic inflammatory disorder of the GI tract that is characterized by mucosal ulceration, rectal bleeding, diarrhea, and abdominal pain. UC and $\mathrm{CD}$ are the 2 major diseases in IBD, and worldwide prevalence has been on the rise. IBD is multi-etiological and is driven by immunological disturbances, genetic alterations, and environmental factors such as diet, lifestyle, and socioeconomic development. However, the etiopathogenesis of IBD is not fully understood. Prior to the development of biological medicine, treating IBD was challenging. The conventional therapies for IBD were 5-aminosalicylic acid, corticosteroids, and immunomodulators; surgery was the only available option for patients who were unresponsive to these treatments.

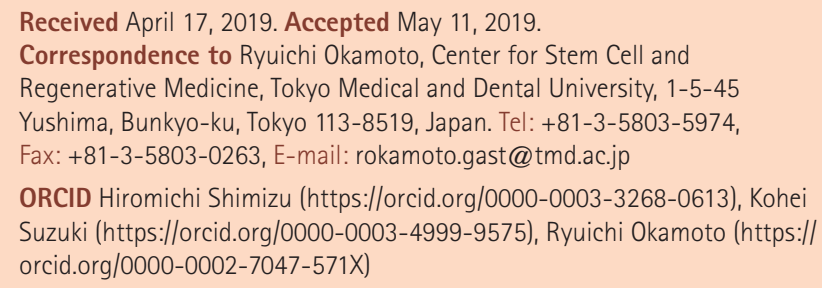

Recently, biological agents, such as anti-TNF- $\alpha$, anti- $\alpha 4 \beta 7$, and anti-interleukin (IL) 12/23 antibodies have been developed and released for IBD treatment. These monoclonal antibody therapies target selective molecules that exacerbate GI damage and have greatly advanced IBD treatment. Treated patients experience complete healing of the intestinal and colonic mucosa. Accumulating evidence suggests that achieving mucosal healing should be the principal aim of IBD treatment as it ameliorates disease outcome in terms of clinical symptoms, disease relapse, hospitalization, and surgeries. ${ }^{1-3}$ However, despite the use of biological agents, we still encounter multidrug resistance in IBD patients which impedes mucosal healing and necessitates surgical intervention. Therefore, it is of paramount importance that an effective therapy is developed for facilitating the achievement of mucosal healing and avoiding potentially invasive surgeries in refractory IBD patients. In the current paper, we review a "stem cell-based therapy" as a promising regenerative treatment for IBD.

\section{MECHANISM OF MAINTAINING GI HOMEOSTASIS}

The GI tract is one of the largest organs in humans, which en- 


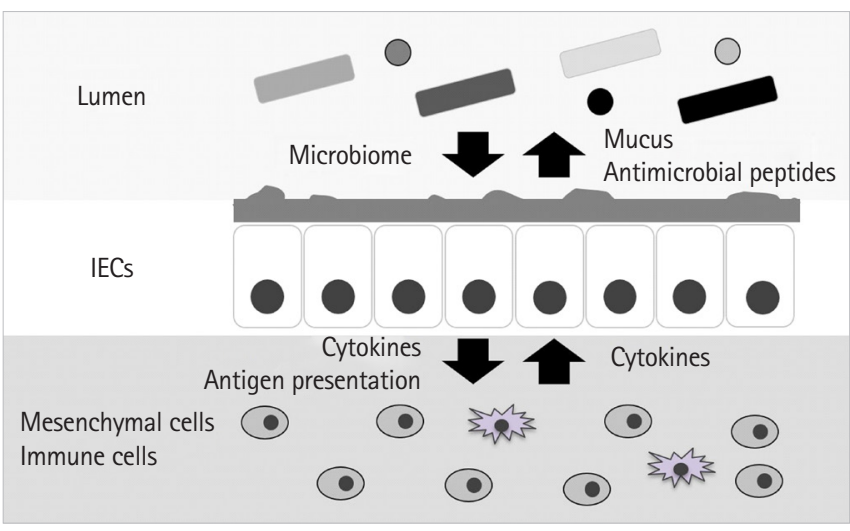

Fig. 1. Intestinal epithelial cells (IECS) are the first barrier against outer environment. The gastrointestinal tract, formed of an IEC monolayer, interacts with the commensal microbiome and protects the organism from pathogenic microbes by secreting antimicrobial peptides and mucins. Further, IECs coordinate with stromal cells, including immune cells and mesenchymal cells, via antigen presentation and cytokine-mediated signaling, to maintain gastrointestinal homeostasis.

counters an external environment with the largest surface area. In addition to being a barrier, it also performs various functions such as digestion and assimilation of food, and secretion of digestive juices and hormones. Moreover, the GI tract is one of the biggest immunological organs that closely interacts with commensal microbiome and protects the organism from pathogenic microbes. The intestinal epithelium comprises a monolayer of intestinal epithelial cells (IECs) that are maintained by intestinal stem cells (ISCs) residing at the bottom of the crypts (Fig. 1). Studies have identified that Lgr5positive crypt base columnar cells function as ISCs, which are also marked by ASCL2, SMOC2, and OLFM4 genes. Another potential ISC subset, called the quiescent ISCs, lack rapid turnover, suggesting that they function as an ISC reservoir in the event of ISC damage by radiation or other causes. ISCs give rise to 5 types of mature differentiated cells every 3 to 4 days. These mature differentiated IECs function as enterocytes, goblet cells, enteroendocrine cells, Paneth cells, and Tuft cells, and are involved in various specific activities. In addition, ISCs are supported by a stem cell "niche" maintained by Paneth cells, ${ }^{4}$ myofibroblasts and other stromal cells providing indispensable stimuli, epidermal growth factor, Wnt, and Notch signals. Nonetheless, further studies are necessary to elucidate the precise mechanisms controlling ISC differentiation, proliferation, and the effect of various ISC niche states on ISC behavior.

\section{MECHANISM OF GI INFLAMMATION IN IBD}

The etiology of IBD has not been fully understood, but it is proposed that both dysregulated innate and adaptive immune pathways contribute to the aberrant inflammatory response in the GI tract. ${ }^{5,6} \mathrm{CD}$ has long been considered to be driven by the Th1 response, while UC is associated with the Th2 response. In CD mucosa, macrophage-derived IL-12, IL-18, and TNF- $\alpha$ are overexpressed, which drives the Thl immune response to upregulate IL-2 and IFN-r production. This response is thought to trigger intestinal inflammation. Contrastingly, UC is characterized by increased expressions of IL-5 and IL-13, which are members of the Th2 cytokine family. In addition to the Th1 and Th2 responses, the role of Th17 cells, a subpopulation of inflammatory $\mathrm{T}$ cells that expand in response to pro-inflammatory cytokines, has been the focus of many recent studies. Th17 cells are induced by IL- 6 and transforming growth factor $\beta$ (TGF- $\beta$ ) and produce IL-17A, IL-21, and IL-22. Higher transcript levels of IL-17A have been reported both in CD and UC mucosa, and its expression has been observed by immunohistochemistry in the lamina propria of IBD patients. These dysregulated $\mathrm{T}$ cell responses with the abnormal development of activated T cell subsets could lead to intestinal inflammation by an excessive release of cytokines and chemokines.

Studies have also focused on mucosal immune responses, such as epithelial barrier integrity, the secretion of various antimicrobial peptides, innate microbial sensing, and programmed cell deaths including apoptosis and necroptosis. In patients with IBD, decreased epithelial barrier function and increased epithelial permeability have been observed. IL-13 released by Th2 cells can exert some potent effects on IECs, such as increasing epithelial permeability and inducing IEC differentiation and apoptosis. A defective expression of protective mucus and antimicrobial peptides in CD patients has also been observed. This could be more pronounced in patients with variant NOD2 whose polymorphisms have shown a strong association with ileal CD and Paneth cell dysfunction.

\section{MECHANISM OF MUCOSAL REGENERATION IN IBD}

The reestablishment of homeostasis in damaged gut mucosa depends on the proper organization of ISCs and IECs. Limited information on the underlying mechanism is available; however, tissue repair and regeneration of damaged mucosa can be divided into the following 3 phases: $^{7}(1)$ Restitution of IECs: IEC migration over damaged and ulcerated areas is the very 
first step of regeneration. IECs migrate over the wound bed in a non-proliferative manner from crypts adjacent to the area. TGF- $\beta$, intestinal trefoil factor, and trefoil factor have been reported to promote this process. $^{8}(2)$ Proliferation of IECs: the second phase involves supplying IECs to cover the wound bed. ISCs in the crypts adjacent to the wound bed are predominantly proliferative, forming channel-like structures to supply IECs to the wounded area. (3) Reconstitution of gut tissue: the channel-like structures invaginate to form multiple fissions from which new crypts arise in the wounded area. During the reconstitution of crypt structure, Wnt5a, part of the noncanonical Wnt signaling generated from myofibroblast in the wound bed, plays an indispensable role. It is also reported that vascular endothelial growth factor secreted by the stromal cells contributes to tissue regeneration. ${ }^{9}$ Yui et al. ${ }^{10}$ reported that IECs have the plasticity to temporarily gain a gene expression pattern similar to that of fetal IECs during gut tissue regeneration, possibly due to the stromal cells in the wound site. Sato et al. ${ }^{11}$ reported that an IEC subpopulation, Dll1-positive IEC secretory progenitor cells, are able to gain plasticity to function as ISCs to contribute to IEC regeneration when ISCs are eliminated by lethal radiation in mice. Similar to this phenomenon, we have previously reported that Atoh1 positive cells, a secretory progenitor cell, are able to function as ISCs for tissue repair in mice with induced colitis. ${ }^{12}$ These emerging reports demonstrate that IECs share their plasticity to serve as ISCs and contribute to tissue regeneration. Thus, ISCs play a crucial role in gut tissue regeneration. The proper spatial allocation of both ISCs and stromal cells, which coordinate with each other for tissue regeneration, is essential.

In recent years, the development of IEC primary culture systems has allowed deeper and more thorough investigations into IECs and ISCs. ${ }^{11}$ We have successfully generated "organoids," cultured primary IECs, from biopsy samples of the small intestine of an IBD patient, and analyzed the gene expression pattern of organoids at a single-cell level. Organoids from the lesion of CD patients had a higher expression of ISC genes and were characterized by faster proliferation and a higher "organoid reconstitution" capacity. These characteristics demonstrate that ISCs in CD patients are predominantly proliferative and regenerative. ${ }^{13}$

\section{STEM CELL-BASED THERAPY IN IBD}

The development of biological agents has greatly improved the treatment of IBD, enabling significant mucosal healing, which is the ultimate therapeutic goal for IBD patients. However, there are patients who are refractory to multidrug treatments, and for these patients' novel and innovative approaches are required. "Stem cell-based therapy" is one of those promising therapies and is currently in the stages of development and clinical trials. The 3 sources of stem cells are as follows: embryonic (ES cell), induced pluripotent (iPS cell), and somatic stem cells. Both ES and iPS cells are currently difficult to implement in therapy due to ethical issues for the former and the potentially oncogenic characteristics of the latter. Research is underway to clarify and mitigate the oncogenic potential of iPS cells. The somatic stem cells, which are found throughout the body in various organs and tissues after development, have less multipotency or multi-differentiative potential than ES and iPS cells. Somatic stem cells multiply by cell division both to self-renew and to generate every cell type of the local organ from which they originate. Unlike ES cells, somatic stem cells are derived from adult tissue, which alleviates the ethical controversy. Further, somatic stem cells do not require any genetic reprogramming, reducing the oncogenic risk for therapeutic use. Therefore, somatic stem cells present numerous advantages as a stem cell source for "stem cell-based therapy." Stem cells can either be autologous or allogeneic, obtained from the patient or a donor, respectively. To date, 2 "stem cell-based therapies" using either hematopoietic stem cells (HSCs) or mesenchymal stem cells (MSCs) have been trialed on IBD patients.

HSCs give rise to a variety of blood cells including both myeloid and lymphoid lineage cells. HSC transplantation (HSCT) has been suggested to reset the immune system, attenuating the abnormal inflammatory immune response in IBD patients. Based on this model, several reports have tried to demonstrate the efficacy of HSCT in moderate to severe $\mathrm{CD} .{ }^{14} \mathrm{HSCT}$ involves resetting the primary immune system through lymphoablation using chemotherapy followed by the reconstitution of the immune system. In this context, autologous HSCT may not adequately address patient predisposition, and although allogenic HSCT may be more appropriate and effective, there is a much greater risk of treatment-related complications such as graft versus host disease. Majority of the reports available pertain to allogeneic HSCT conducted on patients with UC or CD as a treatment for hematological malignancy. Only one randomized control trial using autologous HSCT has been conducted on refractory CD patients. ${ }^{15}$ Of the 45 patients who underwent cell mobilization and randomization, 23 received HSCT while 22 received the standard CD treatment. In the 12 months after autologous HSCT, $38.1 \%(P=0.01)$ more patients 
in the HSCT group were able to stop immunosuppressive medicine compared with the control group. In an imaging study, 25.7\% $(P=0.54)$ more patients in the HSCT group were free from the active disease, and $25.7 \%(P=0.52)$ more patients had a CDAI less than 150 for the last 3 months. CDAI decreased from 326 at baseline to 150.7, compared with 354 to $63.0(P=$ 0.04 ) in the control group. However, this HSCT trial did not result in a statistically significant improvement in sustained disease remission at the end of 1 year. Furthermore, frequent serious adverse events (SAEs) had occurred throughout this trial. Seventy-six SAEs occurred in the HSCT group, while 38 occurred in the control group, and 1 patient who experienced an SAE in the HSCT group died. Almost all patients experienced non-SAEs, 265 events in the HSCT group and 134 in the control group. Thus, the study concluded that the use of HSCT, compared with conventional therapy, was not an acceptable treatment option for patients with refractory $\mathrm{CD} .^{15-17}$

MSCs are derived from the bone marrow, umbilical cord and adipose tissue. They retain pluripotency, transforming into adipocytes, osteoblasts, myocytes, and chondroblasts. MSCs have been reported to have an immunosuppressive effect by polarizing anti-inflammatory M2 macrophages and reducing both dendritic cells and neutrophils through prostaglandin $\mathrm{E}_{2}$ (PGE2) and IL-10 at the wound site. ${ }^{18}$ MSCs can also reduce the activity of T lymphocytes and B lymphocytes by modulating their proliferation and differentiation. ${ }^{19,20}$ There have been several phase I/II clinical studies for both autologous and allogeneic MSC transplantation (MSCT). Bone marrow-derived MSCT (BM-MSCT) has been much safer than HSCT. Although autologous BM-MSCT did not demonstrate a significantly higher efficacy than conventional therapy, allogeneic BM-MSCT seemed to be efficient for luminal CD patients in a phase II multicenter clinical trial. In this trial, CDAI decreased by 167 points $(P<0.0001), 53 \%$ of patients had clinical remission (CDAI $<150)$, and endoscopic improvement was observed in $47 \%$ of patients at 42 days after transplantation. Only one SAE was reported, which was a colonic adenocarcinoma. ${ }^{21}$ Clinical studies have also been conducted using adipose-derived mesenchymal stem cells (ASCs) for CD patients with fistulas. Panés et al. ${ }^{22}$ completed a phase III randomized, double-blind study using allogeneic ASC transplantation (ASCT) for CD patients with complex perianal fistulas. This multicenter clinical study was performed with 212 patients randomly assigned to either the ASCT or placebo group (107 patients vs. 105 patients). At week 24, 50\% of ASCT-treated patients achieved remission, compared with $34 \%$ of the placebo group
$(P=0.024)$. ASCT was found to be safe with similar adverse events to those of the placebo group, which were mostly anal abscesses and proctalgia. ${ }^{21}$ Their extensional study at 52 weeks found that ASCT achieved remission for $59.2 \%$ of the treated patients, compared with $41.6 \%$ of placebo-treated patients $(P=0.013)$. ASCT has been shown to be significantly effective for CD patients with complex perianal fistulas, with adverse effects similar to those for the placebo group. ${ }^{23}$

\section{ISCS FOR STEM CELL-BASED THERAPY}

Thus, stem cell-based therapy could be a promising treatment option for IBD patients refractory to conventional therapies. Presently, MSCT seems highly promising, especially for treating fistulizing and perianal disease, but seems less promising for treating luminal disease. Clinical studies using ASCT showed encouraging results in which more than $50 \%$ of $\mathrm{CD}$ patients with fistulas achieved remission 1 year after transplantation. Contrastingly, HSCT cannot be recommended because of frequent SAEs, even though clinical studies using HSCT showed efficacy to some extent. Currently, we are investigating a novel stem cell-based therapy. Using the IEC culture system, Yui et $\mathrm{al}^{24}$ generated mouse-derived organoids and transplanted ISCs into mice with colitis. They demonstrated the efficacy of ISC transplantation in the treatment of mouse colitis by ISC engraftment on the wound bed and the reconstitution of the damaged tissue. Based on this, we focus on ISC transplantation (ISCT) as one of the possible therapeutic tools for refractory IBD patients. In this study, we derived organoids from bi-

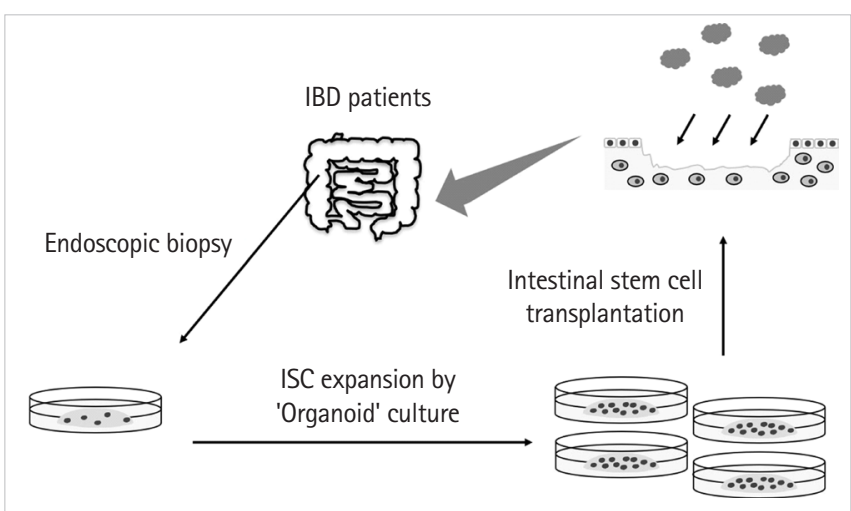

Fig. 2. Autologous intestinal stem cell (ISC) transplantation on IBD patients. Organoids are generated from biopsy samples taken through colonoscopy of an IBD patient, and further cultured and expanded for transplantation on the wound bed of the same patient. Transplantation is planned to be through colonoscopy. 
opsy samples obtained through colonoscopy of an IBD patient and generated enough ISCs for transplantation on the wound bed of the same patient (Fig. 2). This autologous ISCT could potentially cover the wound by supplying both proliferative ISCs and functional IECs to the site (IEC restitution and proliferation). Subsequently, mucosal barrier integrity would be reestablished, protecting the wound bed from both chemical and mechanical stimulations and from luminal pathogenic microorganisms (reconstitution). Hopefully, the local immune abnormality would be corrected through this process, leading to reduced inflammation, although this should be studied further. ISCT is an innovative form of "stem cell-based therapy" that directly addresses mucosal healing by facilitating epithelial restitution, proliferation, and reconstitution. Although the clinical trial is still pending, we are optimistic that ISCT will prove to be a useful therapeutic option for IBD patients refractory to multiple therapies.

\section{CONCLUSIONS}

The achievement of mucosal healing is important for IBD treatment. To improve the treatment paradigm, it is necessary to deepen our understanding of IBD etiology and to investigate new therapeutic methods. "Stem cell-based therapy" is one of the promising new treatment options for refractory IBD patients.

\section{FINANCIAL SUPPORT}

R.O. received the grant of Ministry of Education, Culture, Sports, Science and Technology/Japan Society for the Promotion of Science (MEXT/JSPS) KAKENHI (26221307, 16H05284, 226221307, 18K07934, 18K15743, 18K15774), the Research Center Network Program for Realization of Regenerative Medicine from Japan Agency for Medical Research and Development (AMED) (16bm0304001h0004, 17BM03041H0005, 18bm03041h0006, 19bm0304001h0007).

\section{CONFLICT OF INTEREST}

No potential conflict of interest relevant to this article was reported.

\section{AUTHOR CONTRIBUTION}

Conception and drafting of manuscript: Shimizu H, Suzuki K,
Watanabe M, Okamoto R. Writing - original draft: Shimizu H, Okamoto R. Writing - review and editing: Shimizu H, Suzuki K, Watanabe M, Okamoto R. Approval of final manuscript: all authors.

\section{ACKNOWLEDGEMENTS}

Authors would like to thank Hady Yuki Sugihara, a technician in Center for Stem Cell and Regenerative Medicine, for his help with English editing and manuscript preparation.

\section{REFERENCES}

1. Frøslie KF, Jahnsen J, Moum BA, Vatn MH; IBSEN Group. Mucosal healing in inflammatory bowel disease: results from a Norwegian population-based cohort. Gastroenterology 2007; 133:412-422.

2. Colombel JF, Rutgeerts P, Reinisch W, et al. Early mucosal healing with infliximab is associated with improved long-term clinical outcomes in ulcerative colitis. Gastroenterology 2011;141: 1194-1201.

3. Rutter M, Saunders B, Wilkinson K, et al. Severity of inflammation is a risk factor for colorectal neoplasia in ulcerative colitis. Gastroenterology 2004;126:451-459.

4. Sato T, van Es JH, Snippert HJ, et al. Paneth cells constitute the niche for Lgr5 stem cells in intestinal crypts. Nature 2011;469: $415-418$

5. Eltzschig HK, Carmeliet P. Hypoxia and inflammation. N Engl J Med 2011;364:656-665.

6. Román J, Planell N, Lozano JJ, et al. Evaluation of responsive gene expression as a sensitive and specific biomarker in patients with ulcerative colitis. Inflamm Bowel Dis 2013;19:221229.

7. Okamoto R, Watanabe M. Role of epithelial cells in the pathogenesis and treatment of inflammatory bowel disease. J Gastroenterol 2016;51:11-21.

8. Mashimo H, Wu DC, Podolsky DK, Fishman MC. Impaired defense of intestinal mucosa in mice lacking intestinal trefoil factor. Science 1996;274:262-265.

9. Manieri NA, Mack MR, Himmelrich MD, et al. Mucosally transplanted mesenchymal stem cells stimulate intestinal healing by promoting angiogenesis. J Clin Invest 2015;125:3606-3618.

10. Yui S, Azzolin L, Maimets M, et al. YAP/TAZ-dependent reprogramming of colonic epithelium links ECM remodeling to tissue regeneration. Cell Stem Cell 2018;22:35-49.

11. Sato T, Vries RG, Snippert HJ, et al. Single Lgr5 stem cells build 
crypt-villus structures in vitro without a mesenchymal niche. Nature 2009;459:262-265.

12. Ishibashi F, Shimizu H, Nakata T, et al. Contribution of ATOH1(+) cells to the homeostasis, repair, and tumorigenesis of the colonic epithelium. Stem Cell Reports 2018;10:27-42.

13. Suzuki K, Murano T, Shimizu H, et al. Single cell analysis of Crohn's disease patient-derived small intestinal organoids reveals disease activity-dependent modification of stem cell properties. J Gastroenterol 2018;53:1035-1047.

14. Oyama Y, Craig RM, Traynor AE, et al. Autologous hematopoietic stem cell transplantation in patients with refractory Crohn's disease. Gastroenterology 2005;128:552-563.

15. López-García A, Rovira M, Jauregui-Amezaga A, et al. Autologous haematopoietic stem cell transplantation for refractory Crohn's disease: efficacy in a single-centre cohort. J Crohns Colitis 2017;11:1161-1168.

16. Jauregui-Amezaga A, Rovira M, Marín P, et al. Improving safety of autologous haematopoietic stem cell transplantation in patients with Crohn's disease. Gut 2016;65:1456-1462.

17. Hawkey CJ, Allez M, Clark MM, et al. Autologous hematopoetic stem cell transplantation for refractory Crohn disease: a randomized clinical trial. JAMA 2015;314:2524-2534.

18. Ren G, Zhang L, Zhao X, et al. Mesenchymal stem cell-mediated immunosuppression occurs via concerted action of che- mokines and nitric oxide. Cell Stem Cell 2008;2:141-150.

19. Aggarwal S, Pittenger MF. Human mesenchymal stem cells modulate allogeneic immune cell responses. Blood 2005;105: 1815-1822.

20. Corcione A, Benvenuto F, Ferretti E, et al. Human mesenchymal stem cells modulate B-cell functions. Blood 2006;107:367372.

21. Forbes GM, Sturm MJ, Leong RW, et al. A phase 2 study of allogeneic mesenchymal stromal cells for luminal Crohn's disease refractory to biologic therapy. Clin Gastroenterol Hepatol 2014;12:64-71.

22. Panés J, García-Olmo D, Van Assche G, et al. Expanded allogeneic adipose-derived mesenchymal stem cells (Cx601) for complex perianal fistulas in Crohn's disease: a phase 3 randomised, double-blind controlled trial. Lancet 2016;388:12811290.

23. Panés J, García-Olmo D, Van Assche G, et al. Long-term efficacy and safety of stem cell therapy (Cx601) for complex perianal fistulas in patients with Crohn's disease. Gastroenterology 2018;154:1334-1342.

24. Yui S, Nakamura T, Sato T, et al. Functional engraftment of colon epithelium expanded in vitro from a single adult Lgr5+ stem cell. Nat Med 2012;18:618-623. 